\title{
Assessment the Correlations of Hormones, Lipid Profiles, Oxidative Stress, and Zinc Concentration in Iraqi Women with Polycystic Ovary Syndrome
}

\author{
Ashwaq Shenta ${ }^{1}$, Khansaa Saud ${ }^{1}$, Ali Al-Shawi*1
}

\begin{abstract}
Background: Polycystic ovary syndrome (PCOS) is a hormonal disorder in women with unknown causes and is the leading cause of infertility in women of reproductive age, presenting a wide range of clinical manifestations worldwide. The objective of study is to compare the correlation between hormones, lipid profile, oxidative stress and Zinc concentration in PCOS patients.

Methods: The present study examined hormone levels (progesterone, prolactin, luteinizing and follicle stimulation hormones (LH and FSH, respectively), antioxidant factors (catalase, glutathione-s- transferase), lipid profiles and zinc concentration of 50 Iraqi women patients' diagnosis with PCOS and 40 healthy women, divided in two age groups of 15-29 and 30-45 years. Body mass index was estimated for two age groups.

Results: The results showed decreasing of catalase, glutathione, and $\mathrm{Zn}$ concentrations with an increase in age. A slightly significant increase in LH and prolactin and decrease in high-density lipoprotein (HDL-C) with an increase in age in the patient group compared to the control group was noted.

Conclusions: Our study demonstrated that some factors (such as family history, genetics, environmental, etc...) could play a role in altering hormone levels, lipid profiles, and antioxidant. Controlling these factors may be useful for reducing the PCOS-associated problems in women's health. Needed extensive studies to assess the correlation with insulin resistant and obesity.
\end{abstract}

Keywords: Hormones disorders, Lipid profile, Polycystic ovary syndrome, Zinc element.

\section{Introduction}

Hormonal balance plays an important role a woman's life and in many diseases caused by hormone disorders, such early menopause, primary ovarian insufficiency, ovarian cancer, and polycystic ovary syndrome (PCOS) (1). Polycystic ovary syndrome is one of the common risk diseases in women during the childbearing period (15-45 years) (2). This syndrome creates serious health problems, such as high blood pressure, type 2 diabetes, ability to become pregnant, and uterine cancer (3). Hence, the causes of PCOS are still unknown and may belong to gene, insulin resistance, environmental factors, and/or inflammation, which are affected by androgen concentrations (4). Nowadays, proper management and pathophysiological understanding of PCOS are very important for controlling and diagnosing PCOS. Understanding PCOS mechanisms will encourage patients to recover their health (5). However, clinical studies have shown the diagnostic criteria of PCOS require more investigations to identify new treatments (6). In addition to insulin resistance, inflammatory factors and hyper androgenemia, oxidative stress also contributes to PCOS related long term risks such as cancer. Likewise, understanding the correlation between oxidative stress and PCOS with other factors may clear the 
mechanism of PCOS risk (7). Many women have PCOS and do not feel it because of the low information and education about the PCOS symptoms (8). In Iraq, women are suffering from PCOS, and many cases have been recorded in different areas every year. Iraqi studies showed that several factors may increase PCOS risk in Iraqi women, such as obesity, genetic factors, environmental changes, family history, and elevated trace elements concentrations (9). Trace elements like Selenium, Copper, Zinc, Manganese, Magnesium, and heavy metals (Cobalt, Cadmium and Lead) presented variant relations with PCOS disease and the levels of trace elements could enhance the oxidative stress prevention (10). An important trace element is Zinc which support the biological functions and helps in normal growth and development of immune system defense (11). The concentration changes of Zinc in PCOS patients caused body disorders and several studies have been showed the correlation between Zinc and PCOS and suggested an extensive study to understand the roles of Zinc element in PCOS development (12). The present study was designed to estimate the hormones levels (progesterone, prolactin, and luteinizing and follicle stimulating hormones, (LH and $\mathrm{FSH}$, respectively), antioxidant factors: catalase (CAT), glutathione-s- transferase (GST), and zinc (Zn) concentration) and lipid profiles for Iraqi women patients with PCOS in the southern area of Iraq, Basrah province, Al-Zubair Hospital.

\section{Materials and methods \\ Ethics approval}

The informed consent was not obtained from study participants because the present study was a retrospective data analysis. The study was approved by the Chemistry department, College of Education for Pure Sciences, University of Basrah, Iraq.

\section{Study design}

The study carried out from February, 2019 to December, 2019 at Al -Zubair hospital (Governorate hospital), Basrah, Iraq. It included total volunteers of 50 women patients' diagnosis with PCOS and 40 healthy women, the age of patients and healthy was ranged between 15 to 45 years and divided into two groups: the first group age (15 to 29 years), involved 18 healthy and 20 patients of PCOS, and the second group age (30 to 45 years), involved 22 healthy and 30 patients of PCOS. The diagnosis of PCOS followed Rotterdam criteria (oligo or an ovulation, ultrasonography (USG) or clinical biochemical signs like hyper androgensim). Questionnaire form was made to collect data from each participant involved (medical history, demographics and gynecological history). The diagnosis of USG made by gynecologist who responsible for operating of ultrasound device. Body Mass Index estimated by dividing body weight $(\mathrm{kg})$ by the square height $\left(\mathrm{m}^{2}\right)$. Lipids, antioxidants and hormones kits were purchased from Biolabo and Roch companies. Zinc kit was purchased from Spectrum Company.

\section{Objectives}

To estimate the hormones, antioxidants, zinc concentration and lipid profile in Iraqi women with/without PCOS, and appraisal the differences for future outcomes. Also compared the correlation between catalase and $\mathrm{LH}$ and between total cholesterol and FSH.

\section{Laboratory procedures}

In the second day of menstrual cycle, $5 \mathrm{ml}$ of fasting venous blood were collected from individuals (patients and healthy). It was clotted and centrifuged for 10 minutes with $3000 \mathrm{rpm}$ the blood serum was kept at $20 \mathrm{C}$, until the day of use whereas the remaining serum was put in gel tubes. The values of CAT, GST, and lipid profile: TC (total cholesterol), TG (triglyceride), VLDL-C (very low-density lipoprotein-cholesterol), LDL-C (low-density lipoprotein-cholesterol) and HDL-C (high-density lipoproteincholesterol) were estimated in blood serum by UV-Visible spectrophotometer (Cecil, Ce7200, Germany). The concentrations of FSH (Follicle stimulating hormone), LH (Luteinizing hormone), Prolactin, Progesterone, and Estradiol were estimated in blood serum by automated method (Cobas e411, Hitachi high technology, Japan). 


\section{Determination of Zinc Concentration}

Followed spectrum company protocol, prepared three tubes: blank tube (one $\mathrm{ml}$ of the reagents), Standard tube ( $1 \mathrm{ml}$ of reagent+ $50 \mu \mathrm{l}$ of standard), sample tube (1 $\mathrm{ml}$ of reagent $+50 \mu$ l of sample), the three tubes were mixed individually and incubated for 5 min at $37{ }^{\circ} \mathrm{C}$. Next, measured the absorbance at $560 \mathrm{~nm}$ and calculated zinc concentration in $(\mu \mathrm{g} / \mathrm{dl})$ followed the equation bellow:

Zinc concentration $(\mu \mathrm{g} / \mathrm{dl})=\left(\frac{\text { Aspecimen }}{\text { Astandard }}\right) \times \mathbf{2 0 0}$

\section{Statistical analysis}

SPSS software was used for analysis all the results were tabulated as mean $\pm \mathrm{SD}$. The statistical significance between groups of the study were find out using unpaired students ttest. The correlation regression analysis by the comparison of parameters among women with PCOS. P-value of less than 0.05 was considered statistically significant.

\section{Results}

The study was designed to two groups of ages (15-29) years involved 18 healthy women as control and 20 PCOS patients, (30-45) years involved 22 healthy women as control and 20 PCOS patients. The demography and laboratory parameters were recorded at Al-Zubair Hospital, Basrah, Iraq. BMI was higher in control than PCOS patients in age group (15-29) years, while lower in control than PCOS patients in age group (30-45) years.

The serum levels of CAT, GST, and Zn concentration in the PCOS patients and control group were arranged according to the age groups described above. Significant decreases in CAT and GST enzyme activities and Zn concentration in PCOS patients as compared to control subjects in all age groups $(p<0.0001)$ were noted. The comparison between different age groups of patients showed no significant differences in CAT and GST activities and $\mathrm{Zn}$ concentrations in patients in all age groups, as showed in Table 1.

Table 1. Values of antioxidant enzymes and zinc concentration in Polycystic Ovary Syndrome patients and control groups, by compared in two age's groups and BMI.

\begin{tabular}{clllll}
\hline $\begin{array}{c}\text { Age } \\
\text { (Years) }\end{array}$ & Groups & CAT $(\mathbf{K} / \mathbf{m l})$ & $\boldsymbol{G S T}(\mathbf{U} / \mathbf{L})$ & $\mathbf{Z n}(\boldsymbol{\mu g} / \mathbf{d l})$ & BMI $\left(\mathbf{k g} / \mathbf{m}^{2}\right)$ \\
\hline \multirow{2}{*}{$\mathbf{1 5 - 2 9}$} & Control $(\mathrm{No}=18)$ & $1.55 \pm 0.058$ & $2.29 \pm 0.228$ & $72.66 \pm 4.24$ & $58.55 \pm 5.04$ \\
& $\begin{array}{l}\text { Polycystic } \\
\text { Patients }(\mathrm{No}=20)\end{array}$ & $0.17 \pm 0.023^{* * * *}$ & $1.64 \pm 0.061^{* * * *}$ & $56.10 \pm 10.58^{* * * *}$ & $56.80 \pm 4.64^{\# \mathrm{NS}}$ \\
\hline \multirow{2}{*}{$\mathbf{3 0 - 4 5}$} & Control $(\mathrm{No}=22)$ & $1.69 \pm 0.088$ & $2.73 \pm 0.27$ & $73.59 \pm 5.058$ & $65.50 \pm 7.46$ \\
& $\begin{array}{l}\text { Polycystic } \\
\text { Patients }(\mathrm{No}=30)\end{array}$ & $0.138 \pm 0.028^{* * * *}$ & $1.49 \pm 0.193^{* * *}$ & $48.46 \pm 12.65^{* * * *}$ & $66.033 \pm 6.73^{\# \mathrm{NS}}$ \\
\hline
\end{tabular}

Values were expressed as mean \pm SD.

$*$ The level of significance between patients and control groups; ***p $<0.0001$

\# The level of significance between patients and control groups in BMI; $p>0.05$; NS= Non Significant

The levels of total cholesterol, triglycerides, very-low density lipoprotein, low density lipoprotein, and high-density lipoprotein (TC, TG, VLDL-C, LDL-C, and HDL-C, respectively) levels between different age groups for both the patient and control groups are presented. There were highly significant differences in serum TC, TG, VLDL-C, and LDL-C levels in the controls in both age groups as compared to the same age group of the patients ( $p<0.0001$ and $<0.001$, respectively). On the other hand, there were no significant differences in serum HDL-C levels in patients in both age groups compared to the same age groups in the controls ( $p>0.05$ ), as showed in Table 2.

The levels of FSH, LH, prolactin, progesterone, and estradiol between different age groups for both patients and control groups are presented. There were highly significant differences in serum LH, prolactin, and estradiol levels in controls in both age groups compared to the same age group of the PCOS patients. On the other hand, there was also a slightly significant difference in serum FSH and progesterone levels in patients in both age groups compared to the corresponding age group in the controls, as showed in Table 3. 
Table 2. Concentration levels of TC, TG, VLDL-C LDL-C and HDL-C in Polycystic Ovary Syndrome patients and control groups, by compared in two age's groups and BMI.

\begin{tabular}{|c|c|c|c|c|c|c|c|}
\hline $\begin{array}{c}\text { Age } \\
\text { (Years) }\end{array}$ & Groups & $\begin{array}{l}\text { TC } \\
\text { (mg/dl) }\end{array}$ & $\begin{array}{l}\text { TG } \\
(\mathbf{m g} / \mathbf{d l})\end{array}$ & $\begin{array}{l}\text { VLDL-C } \\
\text { (mg/dl) }\end{array}$ & $\begin{array}{l}\text { LDL-C } \\
\text { (mg/dl) }\end{array}$ & $\begin{array}{l}\text { HDL-C } \\
\text { (mg/dl) }\end{array}$ & $\begin{array}{l}\text { BMI } \\
\left(\mathbf{k g} / \mathbf{m}^{2}\right)\end{array}$ \\
\hline \multirow{2}{*}{$15-29$} & Control $(\mathrm{No}=81)$ & $164.61 \pm 18.97$ & $96.05 \pm 10.9$ & $19.60 \pm 2.71$ & $132.44 \pm 19.6$ & $51.11 \pm 5.26$ & $58.55 \pm 5.04$ \\
\hline & $\begin{array}{l}\text { Polycystic } \\
\text { Patients }(\mathrm{No}=20)\end{array}$ & $216.35 \pm 35.65^{* *}$ & $124.75 \pm 22.6^{\text {watk }}$ & $24.95 \pm 4.53^{\text {** }}$ & $193.04 \pm 37.31^{\text {*akk }}$ & $48.05 \pm 3.96^{\text {fNS }}$ & $56.80 \pm 4.64^{\ddagger \mathrm{NS}}$ \\
\hline \multirow{2}{*}{$30-45$} & Control $(\mathrm{No}=22)$ & $163.59 \pm 33.41$ & $101.81 \pm 16.72$ & $14.81 \pm 3.36$ & $137.57 \pm 34.09$ & $46.22 \pm 5.01$ & $65.50 \pm 7.46$ \\
\hline & $\begin{array}{l}\text { Polycystic } \\
\text { Patients }(\mathrm{No}=30)\end{array}$ & $248.33 \pm 51.41^{\text {*o*k }}$ & $131.36 \pm 25.1^{\text {wat }}$ & $26.46 \pm 5.41^{\text {sose }}$ & $230.77 \pm 55.2^{* * * * *}$ & $43.50 \pm 3.68^{\text {fNS }}$ & $66.033 \pm 6.73^{ \pm \mathrm{NS}}$ \\
\hline
\end{tabular}

Values were expressed as mean \pm SD.

*The level of significance between patients and control groups; ${ }^{* * *} \mathrm{p}<0.0001,{ }^{* *} \mathrm{p}<0.001 ;{ }^{*} \mathrm{p}>0.05$

"The level of significance between patients and control groups in BMI; $>>0.05$; NS= Non Significant

Table 3. Concentrations levels of FSH, LH, Prolactin, Progesterone and Estradiol in Polycystic Ovary Syndrome patients and control groups, by compared two groups of age and BMI.

\begin{tabular}{|c|c|c|c|c|c|c|c|}
\hline $\begin{array}{c}\text { Age } \\
\text { (Years) }\end{array}$ & Groups & $\begin{array}{l}\text { FSH } \\
\text { (Miu/ml) }\end{array}$ & $\begin{array}{l}\text { LH } \\
\text { (Miu/ml) }\end{array}$ & $\begin{array}{l}\text { Prolactin } \\
\text { (ng/ml) }\end{array}$ & $\begin{array}{l}\text { Progesterone } \\
(\mathrm{ng} / \mathrm{ml})\end{array}$ & $\begin{array}{l}\text { Estradiol } \\
\text { (pg/ml) }\end{array}$ & $\begin{array}{l}\text { BMI } \\
\left(\mathbf{k g} / \mathbf{m}^{2}\right)\end{array}$ \\
\hline \multirow{2}{*}{$15-29$} & $\begin{array}{l}\text { Control } \\
(\mathrm{No}=81)\end{array}$ & $5.93 \pm 0.42$ & $0.81 \pm 0.063$ & $12.48 \pm 0.33$ & $1.25 \pm 0.24$ & $128.22 \pm 6.80$ & $58.55 \pm 5.04$ \\
\hline & $\begin{array}{l}\text { Polycystic } \\
\text { Patients } \\
(\mathrm{No}=20)\end{array}$ & $3.86 \pm 42.94^{*}$ & $15.85 \pm 0.93^{\text {**** }}$ & $27.08 \pm 2.33^{* * * *}$ & $0.56 \pm 0.38^{* * * *}$ & $61.50 \pm 5.06^{* * * * *}$ & $56.80 \pm 4.64^{\text {\#NS }}$ \\
\hline \multirow{2}{*}{$30-45$} & $\begin{array}{l}\text { Control } \\
(\mathrm{No}=22)\end{array}$ & $7.66 \pm 0.86$ & $4.37 \pm 1.49$ & $11.12 \pm 0.42$ & $1.104 \pm 0.18$ & $114.81 \pm 4.37$ & $65.50 \pm 7.46$ \\
\hline & $\begin{array}{l}\text { Polycystic } \\
\text { Patients } \\
(\mathrm{No}=30)\end{array}$ & $3.36 \pm 4.12^{\text {**** }}$ & $13.44 \pm 0.89^{* * * * *}$ & $24.28 \pm 1.74^{* * * * k}$ & $0.83 \pm 0.41^{\text {** }}$ & $30.83 \pm 10.23^{3 * * *}$ & $66.033 \pm 6.73^{\text {\#NS }}$ \\
\hline
\end{tabular}

Values were expressed as mean \pm SD.

$*$ The level of significance between patients and control groups; $* * * \mathrm{p}<0.0001$;

${ }^{* *} \mathrm{p}<0.01 ;{ }^{*} \mathrm{p}<0.05$

\# The level of significance between patients and control groups in BIM; P $>0.05$; NS= Non Significant

The general statically analysis of hormones, antioxidants, lipids and zinc parameters showed in Figure 1. In addition, there were correlations between CAT and LH (low increase in CAT with high increase in LH) and total cholesterol and FSH (high increase in TC with low increase in $\mathrm{FSH}$ ) in both group ages. The correlation was obtained via linear regression analysis (Pearson's correlation coefficient), showed a positive significant correlation in PCOS patients. As showed in Figures 2 and 3. 

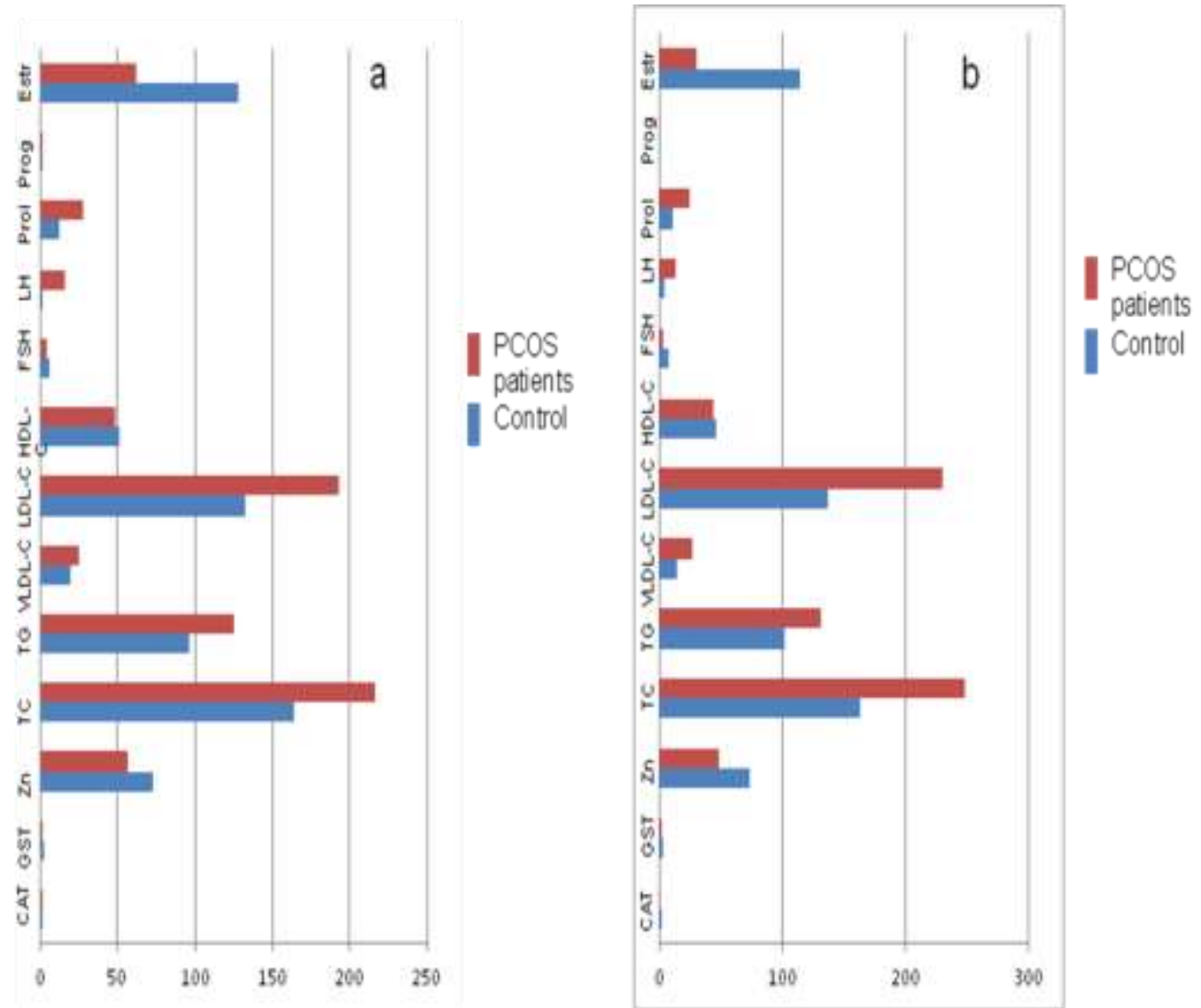

Fig. 1. Statistical analysis of the parameters for: a. Age group (15-29) yeas. b. Age group (30-45) years.

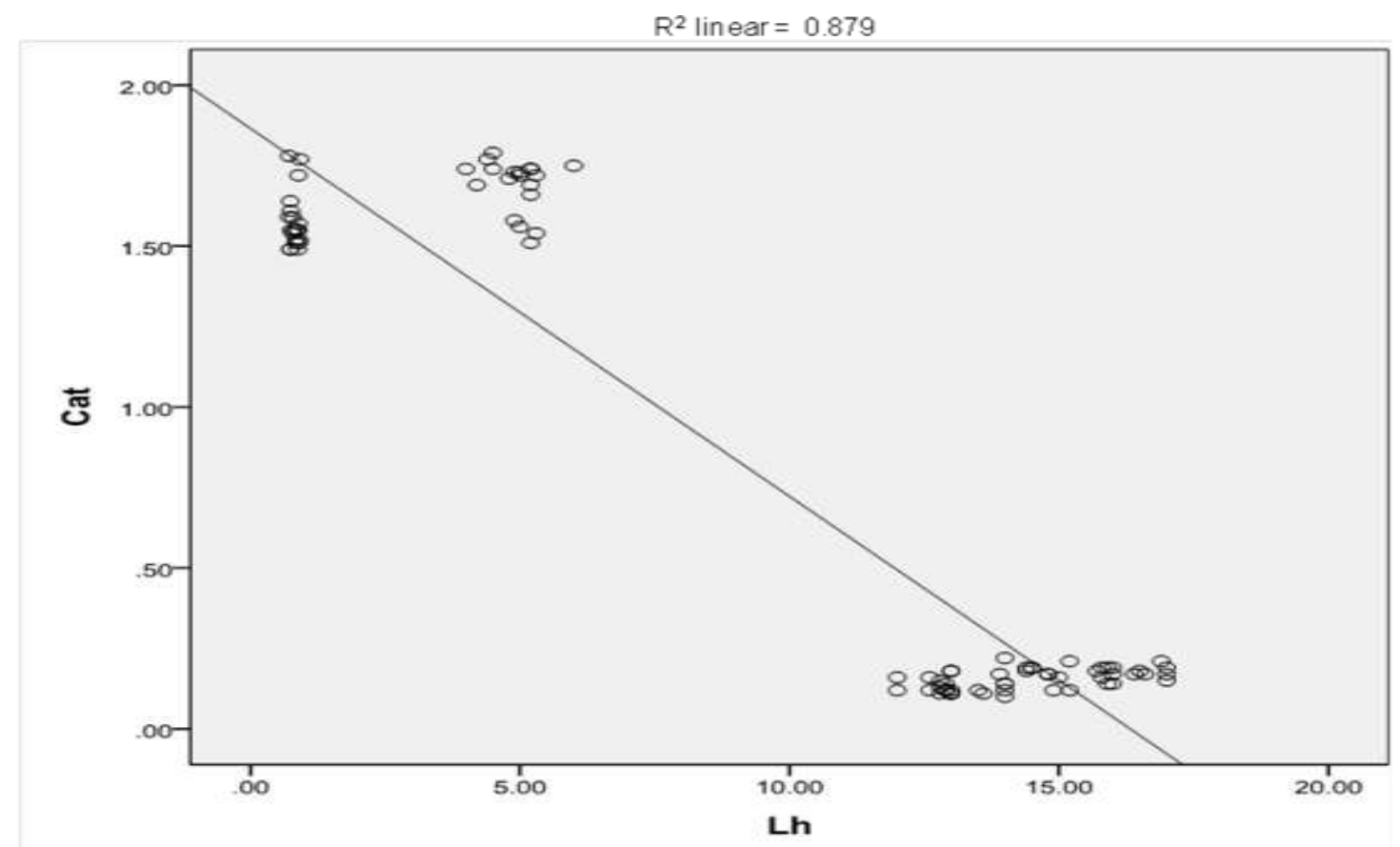

Fig. 2. The correlation between Catalase and LH levels in PCOS groups using linear regression analysis (Pearson correlation). 


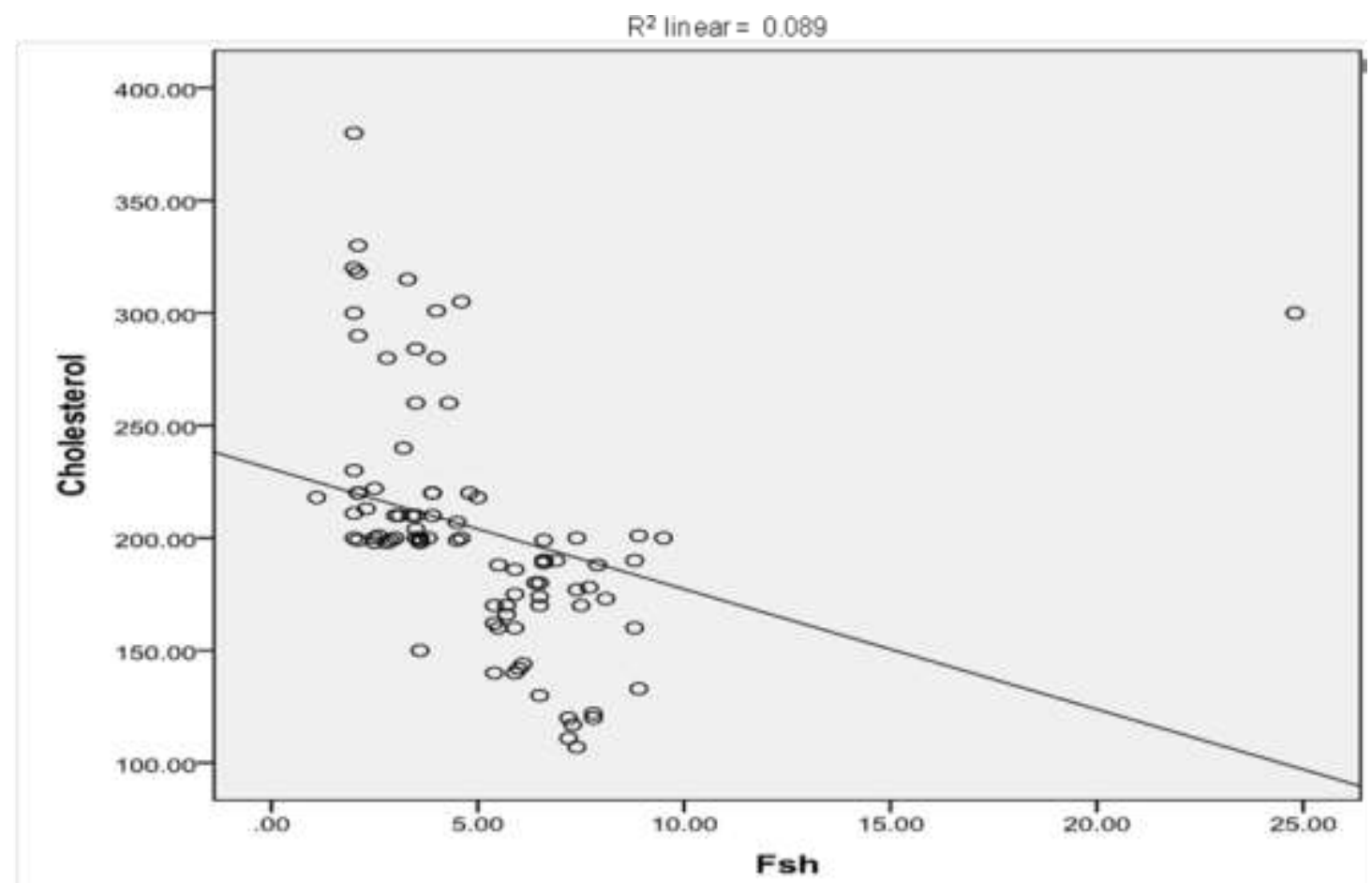

Fig. 3. The correlation between Cholesterol and FSH levels in PCOS groups using linear regression analysis (Pearson correlation).

\section{Discussion}

The increase in hormone levels, lipid profile, and antioxidant factors are higher than normal levels and these elevated levels can disturb the ovarian system and cause PCOS. Hence, the health problems conducted with age could increase and raise the risk of PCOS in women diagnosis with health problems like diabetes, heart attack, stroke, etc., and PCOS maybe increase these health problems. Therefore, early diagnosis and treatment of PCOS patients are important to reduce the risks of health in women. Several Iraqi studies showed the risk of PCOS in women in various areas, Sharqui and colleagues found that free testosterone was positive marker to enhance diagnosis of PCOS (13). Khalaf found that leptin level was associated with endocrinological belongs to fertility (14). Maluki found that PCOS is common factor in women to prevent acne vulgaris (15). Jamil and colleagues found that anti-Mullerian hormone and LH hormones could be predictors for PCOS diagnosis (16). Saeed and his group found that a mutation in the mitochondrial genome and mttRNA could be important factors for PCOS (17). Previous Iraqi studies addressing the treatment of PCOS patients paved the way for more investigations aimed at understanding the PCOS risks and discovering new agents or markers to support women's health. Zinc is one of the important trace elements and play a role in control of follicular, which helps regulate a woman's ovulatory cycle and could improve hirsutism in PCOS patients. The previous studies showed a various levels of zinc in PCOS patients as high or low or no significant levels (11). In this study, the results showed that $\mathrm{Zn}$ concentration decreased in both age groups of PCOS patients compared with the healthy group. In control groups, zinc concentration was slight variance while in PCOS patients in age group (15-29) was higher (zinc concentration $=56.10)$ than age group $(30-45)$ (zinc concentration $=48.46)$. These variances in zinc concentrations in PCOS patients by ages maybe due to some factors could affect patients body such as malnutrition or ignore healthcare. This observation maybe revealed the importance of $\mathrm{Zn}$ in help diagnosis of PCOS associated with antioxidant parameters and require extensive studies with high number of PCOS patients to understand the role of Zinc concentration with age group (30-45) in PCOS patients (18). Several 
studies reported that oxidative stress is significantly higher in PCOS patients than in healthy women (19). Here, the results showed a slightly significant difference in of antioxidant factors, CAT and GST, in PCOS patients compared to healthy women with a decrease corresponding to an increase in age. The estimation was made without considering the obesity factor. The differences in antioxidant factors may belong to the family history and environmental factors of these PCOS patients. Therefore, specific studies should investigate antioxidant factors in numbers of PCOS patients. In this study, lipid profiles showed a significant decrease in VDL-C and HDL-C in both groups in both age ranges, while high significantly levels for TC, TG, and LDL-C were noted. A higher LDL-C to HDL-C ratio revealed a cardiovascular risk to PCOS patients of both age groups. In addition, the hormone profile was observed to have highly significant differences in LH, prolactin, and estradiol levels and insignificant differences in FSH and progesterone between PCOS patients and control groups. The studies showed that the $\mathrm{LH} / \mathrm{FSH}$ ratio is $2-3$-fold different between groups $(20,21)$. In this study, the ratio of LH/FSH in PCOS women was 5:1 for study patients 15-29 years and 4:1 for 30-45 years compared with healthy women ratios in which an elevated LH/FSH ratio could disrupt ovulation. Hence, the positive correlation between CAT and $\mathrm{LH}$ in addition to $\mathrm{TC}$ and $\mathrm{FSH}$ revealed hyperandrogenism in PCOS patients and could be a useful marker for predicting PCOS risk. Likewise, the environmental and genetic factors in

\section{References}

1. Uche A, Angie E, Monica R. Polycystic Ovary Syndrome. A Review of Treatment Options with a Focus on Pharmacological Approaches. P T. 2013;38(6):336-55.

2. Hillman SC, Bryce C, Caleyachetty R, Dale J. Women's experiences of diagnosis and management of polycystic ovary syndrome: a mixed-methods study in general practice. $\mathrm{Br} \mathrm{J}$ Gen Pract. 2020;70(697):e322-e329.

3. Gerard C, Didier D, Evanthia D, F EscobarMorreale H, Franks S, Gambineri A, et al. The polycystic ovary syndrome: a position statement the PCOS patients appeared to have caused variable correlations among the risk factors. However, the increase of age related with health disorders development maybe raise the risk of PCOS develop, therefore, record the full family history of PCOS patients involved health risks disorders before and after PCOS is very important to understand the development of PCOS in women. In addition, observed BMI in age group (15-29) lower than age group (30-45) for both control and PCOS patients, while BMI in control of age group (15-29) was higher than PCOS patients, and in age group (30-45), BMI was lower than PCOS patients. This relation between BMI and variances in ages groups of PCOS patients required further investigation.

In hormone profiles, LH showed a five- fold change over FSH in PCOS patients compared to healthy control values. In the antioxidant profile, $\mathrm{Zn}$ concentration was decreased with age increased. In the lipid profiles, elevations in lipid parameters in PCOS patients could lead to cardiovascular disorders. PCOS risk in women in the age range $30-45$ years were greater than in the 15-29-year-old group. Further studies needed to investigate insulin resistance relation with zinc concentration and obesity of PCOS patients for age group (30-45).

\section{Acknowledgment}

The authors are thankful to Al-zubair hospital (Governorate hospital), Basrah, Iraq, for providing the volunteers of healthy and PCOS patients and laboratory services.

from the European Society of Endocrinology. Eur J Endocrinol. 2014;171(4):P1-29.

4. Tracy L, Ann J. Polycystic Ovary Syndrome: Update on Diagnosis and Treatment. Am J Med. 2014;127(10):912-9.

5. Ana L, Flávia R, Rosana C, Virginia A, Thais M, Ana L. Recent advances in the understanding and management of polycystic ovary syndrome. F1000Res. 2019;8:F1000 Faculty Rev-565.

6. Julian H, Julian H, Ephia Y, Adam B. The diagnosis of polycystic ovary syndrome: The criteria are insufficiently robust for clinical 
research. Clin Endocrinol (Oxf). 2007;67(6):811-5. 7. Tao Z, Minghui Z, Wen XU. Roles of Oxidative Stress in Polycystic Ovary Syndrome and Cancers. Oxid Med Cell Longev. 2016;2016:8589318.

8. Alsaadi YL, Mohamad BJ. Prevalence of hyperandrogenism in Iraqi women with polycystic ovary syndrome. Journal of Science. 2019;60(12):2600-2608.

9. Al-Obaidi MT, Ali ZH, Al-Saadi WI, Al-Wasiti EAR, Al-Aubaidy H. Impact of letrozole versus clomiphene citrate on endometrial receptivity in Iraqi women with polycystic ovarian syndrome. J Clin Pharm Ther. 2019;44(4):618-622.

10. Poli M, Sheila B, Vitor C, Patrícia K, Lívio A. Blood Trace Element Concentrations in Polycystic Ovary Syndrome: Systematic Review and Metaanalysis. Biol Trace Elem Res. 2016.

11. Maryam A, Ehsan G, Amir H, Hamed M, Reza A. Zinc status and polycystic ovarian syndrome: A systematic review and meta-analysis. J Trace Elem Med Biol. 2019;52:216-221.

12. Jamilian M, Foroozanfard F, Bahmani F, Talaee

R, Monavari M, Asemi Z. Effects of Zinc Supplementation on Endocrine Outcomes in Women With Polycystic Ovary Syndrome: A Randomized, Double-Blind, Placebo-Controlled Trial. Biol Trace Elem Res. 2016;170(2):271-8.

13. Sharquie KE, Al-Bayatti AA, Al-Ajeel AI, AlBahar AJ, Al-Nuaimy AA. Free testosterone, luteinizing hormone/follicle stimulating hormone ratio andpelvic sonography in relation to skin manifestations in patients with polycystic ovary syndrome. Saudi Med J. 2007;28(7):1039-43.

14. Khalaf BH. Leptin levels in relation to marital status and neuroendocrine function in Iraqi females with polycystic ovary syndrome. Saudi PharmJ. 2010;18(1):41-4.

15. Maluki AH. The frequency of polycystic ovary syndrome in females with resistant acne vulgaris. $\mathbf{J}$ Cosmet Dermatol. 2010;9(2):142-8.

16. Jamil AS, Alalaf SK, Al-Tawil NG, AlShawaf T. Comparison of clinical and hormonal characteristics among four phenotypes of polycystic ovary syndrome based on the Rotterdam criteria. Arch Gynecol Obstet. 2016;293(2):447-56.

17. Saeed NH, Hamzah IH, Al-Gharrawi SR. Polycystic ovary syndrome dependency on mtDNA mutation; copy Number and its association with insulin resistance. BMC Res Notes. 2019;12(1):455. 18. Abedini M, Ghaedi E, Hadi A, Mohammadi H, Amani R. Zinc status and polycystic ovarian syndrome: A systematic review and meta-analysis. J Trace Elem Med Biol. 2019;52:216-221.

19. Zhang J, Bao Y, Zhou X, Lian Z. Polycystic ovary syndrome andmitochondrial dysfunction. Reprod Biol Endocrinol. 2019;17(1):67.

20. Nath CK, Barman B, Das A, Rajkhowa $\mathrm{P}$, Baruah P, Baruah M. Prolactin and thyroid stimulating hormone affecting the pattern of LH/FSH secretion in patients with polycystic ovary syndrome: A hospital- based study from North East India. J Family Med Prim Care. 2019;8(1):256-260.

21. Le MT, Le VS, Le DD, Nguyen VH, Chen C, Cao NT. Exploration of the role of anti-Mullerian hormone and LH/FSH ratio in diagnosis of polycystic ovary syndrome. Clin Endocrinol (Oxf). 2019;90(4):579-585. 